\title{
Maternal choline status during pregnancy, but not that of betaine, is related to antenatal mental well-being: the GUSTO cohort
}

\author{
L. van Lee ${ }^{1}$, A. Rifkin-Graboi ${ }^{1}$, P.L. Quah ${ }^{1}$, S.M. Saw ${ }^{2}$, F. Yap ${ }^{3}$, K.M. Godfrey ${ }^{4}$, \\ P.D. Gluckman ${ }^{5}$, Y.S. Chong ${ }^{1,6}$, M. Meaney ${ }^{1,7}$, H. Chen ${ }^{3}$ and M.F. Chong ${ }^{1,2}$ \\ ${ }^{1}$ Singapore Institute for Clinical Science, Agency for Science, Technology and Research, Singapore, \\ ${ }^{2}$ Saw Swee Hock School of Public Health, National University of Singapore, Singapore, \\ ${ }^{3} \mathrm{KK}$ Women's and Children's Hospital, Singapore, \\ ${ }^{4}$ Medical Research Council Lifecourse Epidemiology Unit Biomedical Research Centre, University and NIHR \\ Southampton of Southampton and University Hospital Southampton NHS Foundation Trust, Southampton, UK, \\ ${ }^{5}$ Liggins Institute, University of Auckland, New Zealand, \\ ${ }^{6}$ Department of Obstetrics \& Gynaecology, Yong Loo Lin School of Medicine, National University of Singapore and \\ National University Health System, Singapore, Singapore and \\ ${ }^{7}$ Departments of Psychiatry and Neurology \& Neurosurgery, McGill University, Montreal, Canada
}

Choline and betaine status have previously been associated with depression symptoms. No studies to date have examined the influence of maternal choline and betaine status during pregnancy on antenatal and postnatal mental well-being, yet this may be important as pregnant women have a higher dietary choline requirement to meet the needs of the growing fetus ${ }^{(1)}$.

Maternal plasma choline and betaine concentrations $(\mu \mathrm{mol} / \mathrm{L})$ were measured at 26-28 weeks gestation in the GUSTO motheroffspring cohort. Participants filled out the State-Trait Anxiety Inventory (STAI) and Edinburgh Postnatal Depression Scale (EDPS) at 26-28 weeks gestation $(n=949)$ and at 3 months postnatal $(n=689)$, where higher scores were indicative of more depression and anxiety symptoms. Multivariate linear regression models were used to estimate the association of choline and betaine with ante- and postnatal mental well-being, adjusting for covariates.

The mean (SD) antenatal plasma choline and betaine concentrations were $9 \cdot 2(1 \cdot 6) \mu \mathrm{mol} / \mathrm{L}$ and $13 \cdot 1(2 \cdot 7) \mu \mathrm{mol} / \mathrm{L}$, respectively Plasma choline concentrations were positively associated with antenatal depression [ $\beta$ 0.24 EPDS score (95\% CI 0.05, 0.43) per $\mu \mathrm{mol} / \mathrm{L}]$ and anxiety scores $[\beta 0.46 \mathrm{STAI}-$ state score $(95 \% \mathrm{CI} 0 \cdot 03,0.88)$ per $\mu \mathrm{mol} / \mathrm{L}]$, upon adjusting for covariates. Plasma betaine concentrations were not associated with antenatal depression or anxiety. No associations were observed between choline, betaine, and postnatal mental well-being.

This study is the first to report that higher maternal plasma choline status is associated with more symptoms of antenatal depression and anxiety, while plasma betaine concentrations showed no associations. No associations were observed for postnatal mental wellbeing. Prospective studies are required to replicate these findings and further examine the direction of causality and possible biological mechanisms. This study is part of a series of work examining maternal nutrition and mental well-being in the GUSTO study ${ }^{(2,3)}$.

This research is supported by the Singapore National Research Foundation under its Translational and Clinical Research (TCR) Flagship Programme and administered by the Singapore Ministry of Health's National Medical Research Council (NMRC), Singapore- NMRC/TCR/004-NUS/2008; NMRC/TCR/012-NUHS/2014. Additional funding is provided by the Singapore Institute for Clinical Sciences, Agency for Science Technology and Research (A*STAR), Singapore.

1. IOM. Standing Committee on the Scientific Evaluation of Dietary Reference Intakes and its Panel on Folate Other B Vitamins and Choline. Dietary Reference Intakes for Thiamin, Riboflavin, Niacin, Vitamin B6, Folate, Vitamin B12, Pantothenic Acid, Biotin, and Choline. Washington (DC): National Academies Press (US), 1998.

2. Chong MF, Wong JX, Colega M, Chen LW, van Dam RM, Tan CS, et al. Relationships of maternal folate and vitamin B12 status during pregnancy with perinatal depression: The GUSTO study. Journal of psychiatric research. 2014;55:110-6.

3. Chong MF, Ong YL, Calder PC, Colega M, Wong JX, Tan CS, et al. Long-chain polyunsaturated fatty acid status during pregnancy and maternal mental health in pregnancy and the postpartum period: results from the GUSTO study. The Journal of clinical psychiatry. 2015;76(7):e848-56. 